\title{
Rapidly evolving asymptomatic eosinophilia in a patient with lung adenocarcinoma causes cognitive disturbance and respiratory insufficiency: Case report
}

\author{
CHENG-HSIANG LO ${ }^{1}$, YEE-MIN JEN ${ }^{1}$, WEN-CHIUAN TSAI ${ }^{2}$, PING-YING CHUNG ${ }^{3}$ and WOEI-YAU KAO ${ }^{3}$ \\ Departments of ${ }^{1}$ Radiation Oncology and ${ }^{2}$ Pathology; ${ }^{3}$ Division of Hematology and Oncology, \\ Department of Internal Medicine, Tri-Service General Hospital, National Defense Medical Center, Taiwan, R.O.C
}

Received August 27, 2012; Accepted October 31, 2012

DOI: $10.3892 / 01.2012 .1020$

\begin{abstract}
Paraneoplastic eosinophilia is an unusual manifestation that usually remains asymptomatic. In this report, we presented the case of an 82-year-old patient with poorly differentiated lung adenocarcinoma and asymptomatic eosinophilia. The patient's condition worsened rapidly over a week, with episodes of cognitive disturbance, shortness of breath and acute kidney dysfunction. These symptoms were associated with a 4-fold increase in circulating eosinophil counts. The poor condition hindered further anticancer treatment. Treatment of the eosinophilia with corticosteroids and hydroxyurea significantly reduced circulating eosinophil counts to below the initial levels. Results of this case report suggested that lung cancer patients should be monitored closely for rapidly worsening symptoms of cognitive disturbance and respiratory insufficiency as signs of life-threatening asymptomatic eosinophilia, in order to initiate corticosteroid treatment.
\end{abstract}

\section{Introduction}

The development of eosinophilia within solid tumors is a rare manifestation, accounting for $\sim 1 \%$ of all cancer patients (1). Since a number of medical conditions are associated with eosinophilia (2), paraneoplastic eosinophilia is diagnosed by exclusion. Depending on the etiology, the consequences of paraneoplastic eosinophilia may range in severity from asymptomatic to life-threatening. Eosinophilia is usually treated successfully with corticosteroids. Paraneoplastic eosinophilia has been reported in a few cases of lung cancer, including lung squamous cell carcinoma $(3,4)$, non-small-cell

Correspondence to: Dr Woei-Yau Kao, Division of Hematology and Oncology, Department of Internal Medicine, Tri-Service General Hospital, No. 325, Sec 2, Cheng-Gong Road, Neihu, Taipei 114, Taiwan, R.O.C

E-mail:wykao5746@gmail.com

Key words: lung cancer, eosinophilia, paraneoplastic syndrome lung carcinoma (5) and lung adenocarcinoma (6). In the latter case, the patient succumbed rapidly following a tumor relapse associated with rapidly evolving eosinophilia. These studies emphasize the importance of identifying the early signs of aggressive paraneoplastic eosinophilia to initiate corticosteroid treatment prior to end-organ failure.

The transition from asymptomatic to life-threatening paraneoplastic eosinophilia is rapid and difficult to diagnose upon summary examination of the patient, particularly in lung cancer patients who are expected to suffer from respiratory complications. While paraneoplastic eosinophilia is often linked with the overexpression of interleukin (IL)-5 in tumor cells, this type of diagnosis is impractical for such a rapidly evolving and life-threatening complication (4).

In this report, we present a case of paraneoplastic eosinophilia in a patient diagnosed with lung adenocarcinoma. The condition of the 82-year-old male degenerated suddenly, as circulating eosinophil counts increased 4 -fold over a few days. The patient experienced cognitive disturbance and shortness of breath, which may represent new diagnostic tools for early corticosteroid treatment to avoid organ damage. The study was approved by the Ethics Committee of the Tri-Service General Hospital, National Defense Medical Center, Taiwan, R.O.C. Informed consent was obtained from the patient's family.

\section{Case report}

An 82-year-old male was admitted to our hospital on October 5, 2011, with a 2-week history of right-sided flank pain and abdominal fullness. An abdominal sonogram revealed a huge liver mass and the patient was then admitted to our gastro-intestinal (GI) section. The patient had a history of well-controlled chronic obstructive pulmonary disease (COPD), hypertension and benign prostate hyperplasia. The patient had herniorrhaphy 1 year earlier and had received amlodipine, tamsulosin and PRN ipratropium/albuterol turbuhaler. The patient had no known allergies and had smoked half a pack of cigarettes per day for 40 years, after which the patient quit for 20 years.

Laboratory data revealed the following: white blood cells, 52,310 cells $/ \mu 1$ with $46.3 \%$ neutrophils and $45.4 \%$ eosinophils; $13.3 \mathrm{~g} / \mathrm{dl}$ hemoglobin and 242,000 cells/ $\mu$ l platelets; 
A

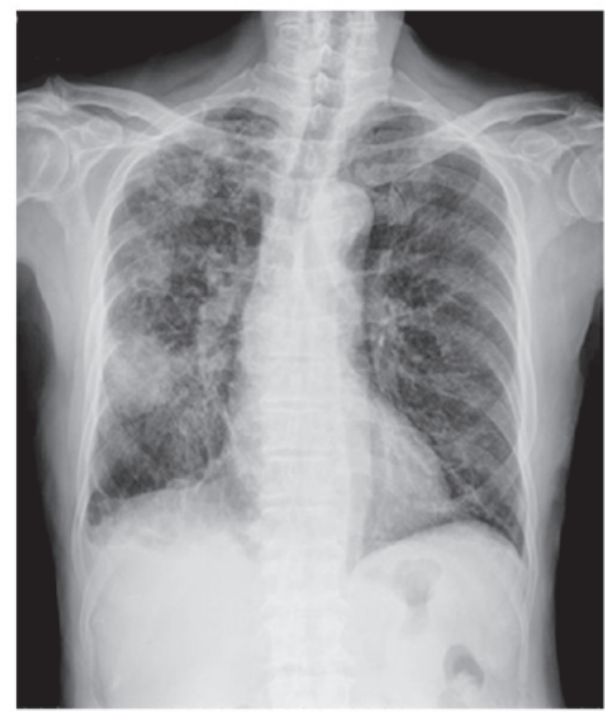

B

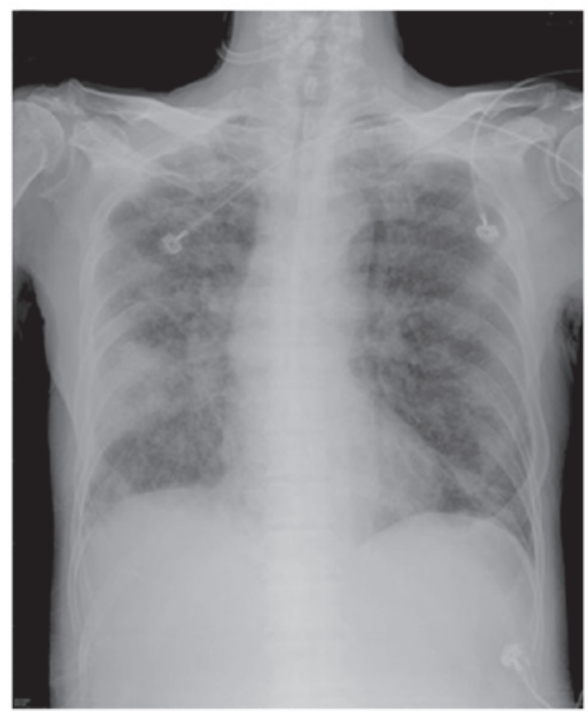

Figure 1. Chest radiograph shows an ill-defined mass lesion $\sim 5 \mathrm{~cm}$ in size at the (A) right middle lung zone at first admission and (B) diffuse infiltration and ground glass opacities over bilateral lung fields outside the mass at readmission.

renal functional insufficiency with $36 \mathrm{mg} / \mathrm{dl}$ blood urea nitrogen (BUN) and $1.4 \mathrm{mg} / \mathrm{dl}$ creatinine; a routine stool test revealed no evidence of parasite infection; immunoglobulin $\mathrm{E}$ level was $99.1 \mathrm{IU} / \mathrm{ml}$ and the levels of tumor markers in the blood, including carcinoembryonic antigen (CEA; $6.47 \mathrm{ng} / \mathrm{ml}$ ) and cancer antigen (CA) 19-9 (49.81 U/ml), were elevated.

On admission, crackles were heard in the right lower lung field. Abdominal palpation revealed mild epigastric tenderness without muscle guarding. A chest radiograph revealed an ill-defined mass lesion $\sim 5 \mathrm{~cm}$ in size in the right middle lung zone (Fig. 1A). Computed tomography of the chest revealed a right middle lung lobe mass and multiple variable-sized nodules in the two lung fields. Computed tomography of the abdomen demonstrated several peripherally enhancing lesions in the lobes of the liver. Magnetic resonance imaging of the brain revealed no evidence of metastasis. Whole-body bone scan revealed multiple bone metastases. Biopsies of the liver and lung mass were performed and pathology revealed poorly differentiated adenocarcinoma of the lung, positive for thyroid transcription factor-1 (TTF-1; Fig. 2A-C). Definitive oral-targeted therapy was advised if epidermal growth factor receptor (EGFR) abnormality was present due to the patient's end stage and old age.

The patient was discharged following completion of the staging work-up and waited for the result of the EGFR analysis. One week later, the patient was readmitted for cognitive disturbance and shortness of breath. On arrival, the patient was noted to be agitated and disoriented and had disorganized speech. Physical examination revealed diffuse wheezing over all lung fields. Pitting edema was noted on the legs. The peripheral white blood cell count had increased 4-fold over a week $(168,800$ cells $/ \mathrm{ml})$, with a proportional increase in eosinophil counts $(55.2 \%)$. Elevated potassium $(5.6 \mathrm{mmol} / \mathrm{l})$, uric acid $(13.8 \mathrm{mg} / \mathrm{dl})$, creatinine $(2.7 \mathrm{mg} / \mathrm{dl})$ and lactate dehydrogenase (LDH; $420 \mathrm{U} / \mathrm{l}$ ) levels were also noted. Chest radiography demonstrated diffuse infiltration and ground glass opacities over the two lung fields in addition to the previous finding (Fig. 1B). Brain computed tomography presented no special findings. Bone marrow biopsy was performed, which revealed reactive bone marrow hypercellularity with a markedly high eosinophil count (Fig. 3A). The average percentage of eosinophils was $39 \%$, compared to $1-5 \%$ in normal bone marrow. Chromosomal analysis demonstrated normal karyotype. Immunohistochemical analysis using the monoclonal mouse anti-human IL-5 antibody (R\&D Systems, Minneapolis, MN, USA) demonstrated that IL-5 was specifically expressed in tumor cells (Fig. 2D). Lung cancer-associated paraneoplastic eosinophilia and acute renal dysfunction were diagnosed.

The patient was treated with hydration and allopurinol to control extreme hyperuricemia. Due to the old age and weak condition of the patient, anticancer treatment was not provided. Since eosinophilia-related organ damage was suspected, hydroxyurea and corticosteroid were administered to reduce the number of eosinophils. The white blood cell count was reduced significantly after 9 days of treatment to 65,500 cells $/ \mu 1$ with $23 \%$ eosinophils (Fig. 3B). The patient's conscious state, kidney function and blood cell count improved following treatment. However, the dyspnea persisted and the patient acquired pneumonia 4 days after the second admission. The family refused further treatment and intervention due to the poor prognosis. The patient succumbed to healthcare-acquired pneumonia with severe sepsis due to Pseudomonas aeruginosa, 10 days after admission.

\section{Discussion}

The present report describes a case of lung adenocarcinoma complicated by severe and aggressive eosinophilia. A number of medical conditions, including allergic disorders, parasitic and fungal infections, vasculitis and drug reactions, as well as hematologic and non-hematologic malignancies are associated with eosinophilia (2). The fact that our patient did not present any of these conditions supports the paraneoplastic nature of the eosinophilia. The pathogenesis of paraneoplastic eosinophilia is unclear. Numerous mechanisms have been 
A

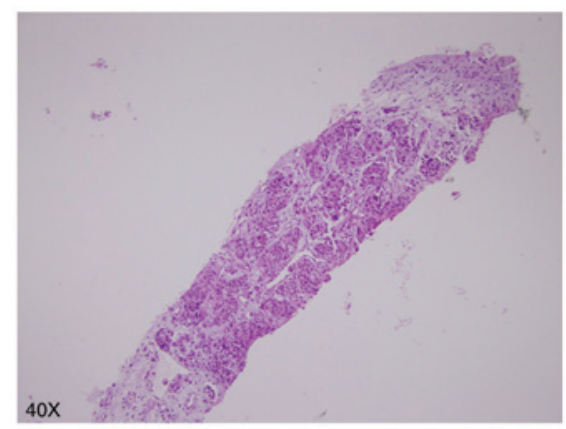

C

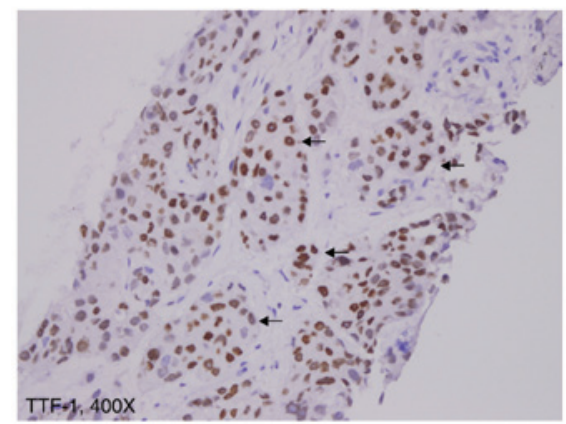

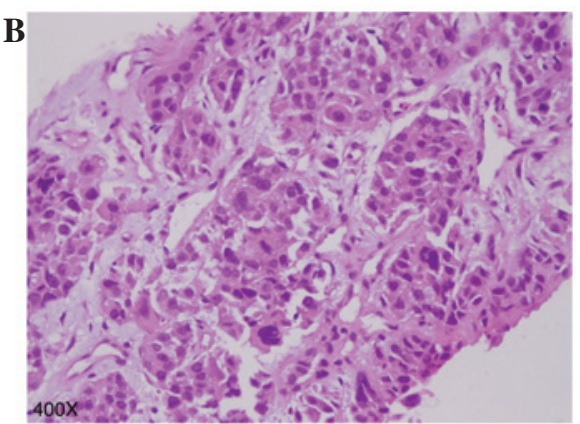

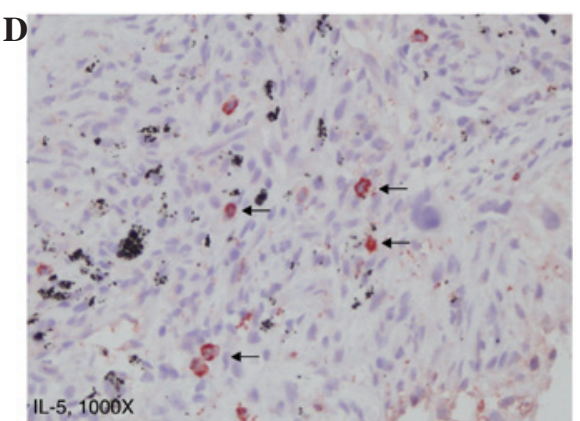

Figure 2. Lung histology showing (A and B) solid nests of tumor cells with nuclear pleomorphism, hyperchromatism and high nuclear/cytoplasmic (N/C) ratios arranged in a solid and focal acinar pattern infiltrating the stroma, which is consistent with poorly differentiated adenocarcinoma. (C) Immunohistochemical staining of lung tumor tissue using thyroid transcription factor-1 (TTF-1) and (D) monoclonal mouse anti-human IL-5 antibody, demonstrating that the tumor is of lung origin and has a specific expression of IL-5 (indicated by arrows).

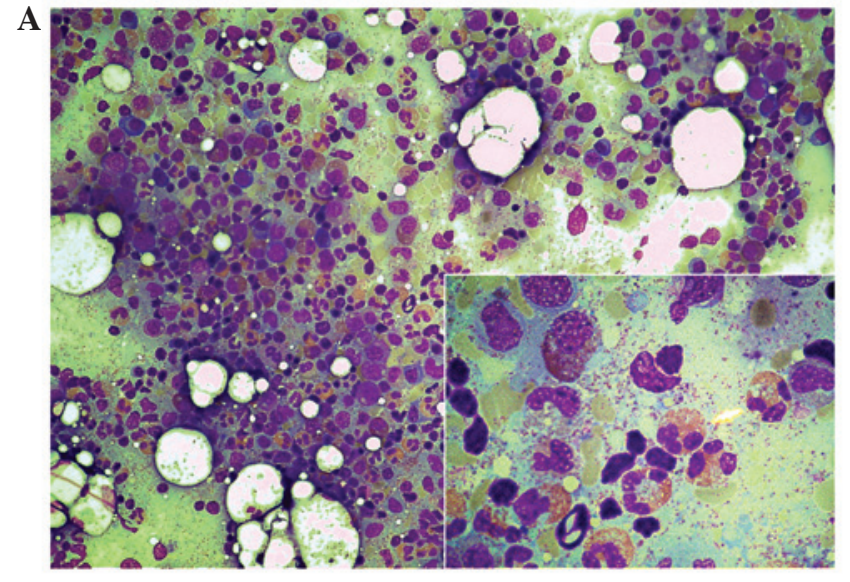

B $\quad$-........ Total leukocyte count

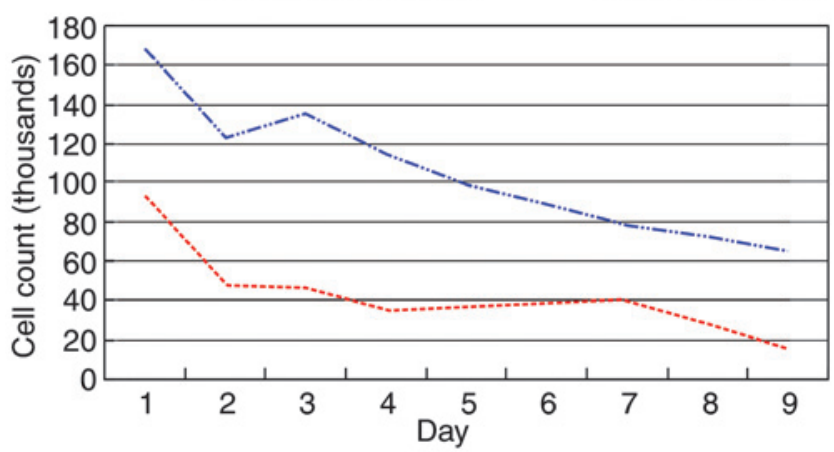

Daily leukocyte and eosinophil count during hospitalization

Figure 3. (A) Reactive bone marrow hypercellularity with high eosinophi counts, which has an average percentage of $>30 \%$, compared to $1-5 \%$ in normal bone marrow. Inset: high-power field shows scattered normal eosinophils. (B) Daily total leukocyte and absolute eosinophil counts were reduced progressively with the combination treatment of hydroxyurea and steroid. postulated and bone marrow stimulation by cytokines secreted by tumor tissues, including granulocyte macrophage-colony stimulating factor (GM-CSF), G-CSF, IL-3 and IL-5, is most commonly reported $(4,7-11)$. In our case, the immunoreactivity of tumor cells to IL-5 is consistent with that reported in these previous studies.

Patients with paraneoplastic eosinophilia are typically asymptomatic. However, in a number of cases, a markedly elevated eosinophil count may be associated with shortness of breath and wheezing. In the present case, the patient exhibited shortness of breath and cognitive disturbance in the form of agitation, disorientation and disorganized speech. Normally, anticancer therapies also resolve the eosinophilia. Matsumoto et al reported a return to normal hematologic status with chemotherapy (12) and Pandit et al demonstrated that leukocytosis and eosinophilia normalize following tumor removal (4).

Primary eosinophilic syndromes are managed successfully with corticosteroid therapy (13-15). However, a number of patients are non-responsive to corticosteroids, but respond well to hydroxyurea (16). Hydroxyurea is also reported to be an effective first-line agent in hypereosinophilic syndrome (15). A combination of hydroxyurea and corticosteroid increases the response rate (15). However, there is no standard treatment for paraneoplastic eosinophilia. To prevent potential harmful effects from chronic exposure of organs to excessive eosinophils, we used a combination of corticosteroid and hydroxyurea, which led to a marked improvement in blood cell counts. The significant effect of corticosteroid and hydroxyurea in reducing the eosinophil count may play a role in improving and stabilizing paraneoplastic eosinophilia and act as a bridge to more anticancer therapies. 
The clinical significance of eosinophilia in cancer patients is undefined. Iwasaki et al report that tumor-associated eosinophilia is associated with a good prognosis (17). However, more studies support the view that paraneoplastic eosinophilia reflects a more extensive disease and poor prognosis (7,18-21). Anagnostopoulos et al suggested that the return of eosinophilia may be an indicator of tumor recurrence (10). In our case, the extremely high eosinophil count and its rapid rise suggested aggressive disease progression and poor prognosis. The addition of combination therapies (corticosteroid and hydroxyurea) to anticancer drugs in paraneoplastic eosinophilia may be beneficial to patient prognosis.

In conclusion, this is the first report of cognitive impairment in combination with respiratory insufficiency as symptoms of rapidly worsening paraneoplastic eosinophilia (eosinophil surge) in cancer patients. This condition may be used for an early diagnosis to initiate corticosteroid treatments and avoid organ damage. This case also suggests that lung cancer patients who present abnormally high counts of eosinophils, should receive a combination of corticosteroids, hydroxyurea and anticancer drugs to prevent the development of aggressive and life-threatening eosinophilia, even if they are asymptomatic initially. This is likely to also enhance the benefits of the anticancer treatment.

\section{References}

1. Jameson JL and Johnson BE: Paraneoplastic syndromes: endocrinologic/hematologic. In: Harrison's Principles of Internal Medicine. Fauci AS, Braunwald E, Kasper DL, Hauser SL, Longo DL, Jameson JL and Loscalzo J (eds). 17th edition. McGraw Hill Medical, New York, NY, pp617-622, 2008.

2. Brito-Babapulle F: The eosinophilias, including the idiopathic hypereosinophilic syndrome. Br J Haematol 121: 203-223, 2003.

3. Zhu YL, Tong ZH, Jin ML and Wang C: Lung cancer with marked blood eosinophilia: case report and literature review. Zhonghua Jie He He Hu Xi Za Zhi 32: 369-372, 2009 (In Chinese).

4. Pandit R, Scholnik A, Wulfekuhler L and Dimitrov N: Non-smallcell lung cancer associated with excessive eosinophilia and secretion of interleukin-5 as a paraneoplastic syndrome. Am J Hematol 82: 234-237, 2007.

5. Verstraeten AS, De Weert A, van Den Eynden G, Van Marck E, Snoeckx A and Jorens PG: Excessive eosinophilia as paraneoplastic syndrome in a patient with non-small-cell lung carcinoma: a case report and review of the literature. Acta Clin Belg 66: 293-297, 2011.

6. Andriamanantena D, Boye T, Gervaise A, Vieu C, Splingard B, Dot JM, Veran Y, et al: An unusual paraneoplastic manifestation in lung cancer: eosinophilic erythroderma. Rev Pneumol Clin 65: 32-35, 2009.
7. Watanabe M, Ono K, Ozeki Y, Tanaka S, Aida S and Okuno Y: Production of granulocyte-macrophage colony-stimulating factor in a patient with metastatic chest wall large cell carcinoma. Jpn J Clin Oncol 28: 559-562, 1998.

8. Sawyers CL, Golde DW, Quan S and Nimer SD: Production of granulocyte-macrophage colony-stimulating factor in two patients with lung cancer, leukocytosis, and eosinophilia. Cancer 69: 1342-1346, 1992.

9. Nakada T, Sato H, Inoue F, Mizorogi F, Nagayama K and Tanaka T: The production of colony-stimulating factors by thyroid carcinoma is associated with marked neutrophilia and eosinophilia. Intern Med 35: 815-820, 1996.

10. Anagnostopoulos GK, Sakorafas GH, Kostopoulos P, Margantinis G, Tsiakos S, Terpos E, Pavlakis G, et al: Disseminated colon cancer with severe peripheral blood eosinophilia and elevated serum levels of interleukine-2, interleukine-3, interleukine-5, and GM-CSF. J Surg Oncol 89: 273-275, 2005.

11. Kato H, Kohata K, Yamamoto J, Ichikawa S, Watanabe M, Ishizawa $\mathrm{K}$, Ichinohasama R, et al: Extreme eosinophilia caused by interleukin-5-producing disseminated colon cancer. Int $\mathrm{J}$ Hematol 91: 328-330, 2010.

12. Matsumoto S, Tamai T, Yanagisawa K, Kawamura S and Fujita S: Lung cancer with eosinophilia in the peripheral blood and the pleural fluid. Intern Med 31: 525-529, 1992.

13. Ogbogu PU, Bochner BS, Butterfield JH, Gleich GJ, Huss-Marp J, Kahn JE, Leiferman KM, et al: Hypereosinophilic syndrome: a multicenter, retrospective analysis of clinical characteristics and response to therapy. J Allergy Clin Immunol 124: 1319-1325, 2009.

14. Tefferi A, Patnaik MM and Pardanani A: Eosinophilia: secondary, clonal and idiopathic. Br J Haematol 133: 468-492, 2006.

15. Gotlib J: World Health Organization-defined eosinophilic disorders: 2011 update on diagnosis, risk stratification, and management. Am J Hematol 86: 677-688, 2011.

16. Srinivasan A, Lavanya R and Sankar J: Steroid-unresponsive hypereosinophilic syndrome. Ann Trop Paediatr 31: 273-277, 2011.

17. Iwasaki K, Torisu M and Fujimura T: Malignant tumor and eosinophils: Prognostic significance in gastric cancer. Cancer 58: 1321-1327, 1986

18. Teoh SC, Siow WY and Tan HT: Severe eosinophilia in disseminated gastric carcinoma. Singapore Med J 41: 232-234, 2000.

19. Chang WC, Liaw CC, Wang PN, Tsai YH and Hsueh S: Tumor-associated hypereosinophilia: report of four cases. Changgeng Yi Xue Za Zhi 19: 66-70, 1996.

20. Reddy SS, Hyland RH, Alison RE, Sturgeon JF and Hutcheon MA: Tumor-associated peripheral eosinophilia: two unusual cases. J Clin Oncol 2: 1165-1169, 1984.

21. El-Osta H, El-Haddad P and Nabbout N: Lung carcinoma associated with excessive eosinophilia. J Clin Oncol 26: 3456-3457, 2008. 